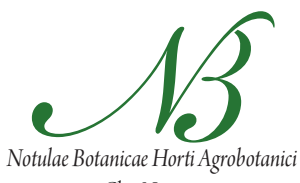

Cluj-Napoca

\title{
Physiological and Biochemical Effects of $\gamma$-Irradiation on Cowpea Plants (Vigna sinensis) under Salt Stress
}

\author{
Hossam S. EL-BELTAGI'**, Heba I.MOHAMED², Abdel Haleem M.A. \\ MOHAMMED², LailaM.ZAKI², Asmaa M.MOGAZY² \\ ${ }^{1}$ Cairo University, Faculty of Agriculture, Biochemistry Dept., P. Box 12613, Gamma st, \\ Giza, Cairo,Egypt; lbltg@yahoo.com (*corresponding author) \\ ${ }^{2}$ Ain Shams University, Faculty of Education, Biological and Geological Sciences Department, El Makres St. Roxy, Cairo 1575, Egypt
}

\begin{abstract}
Soil salinity is one of the most severe factors limiting growth and physiological response in cowpea plants. In this study, the possible role of $\gamma$-irradiation in alleviating soil salinity stress during plant growth was investigated. Increasing salinity in the soil $(25,50,100$ and $200 \mathrm{mM} \mathrm{NaCl}$ ) decreased plant growth, photosynthetic pigments content, total carbohydrate content and mineral uptake compared to control, while increased total phenol content, proline, total free amino acids and lipid peroxidation. Seed irradiation with gamma rays significantly increased plant growth, photosynthetic pigments, total carbohydrate, total phenol, proline, total free amino acids and the contents of $\mathrm{N}, \mathrm{P}, \mathrm{K}^{+}, \mathrm{Ca}^{+2}$ and $\mathrm{Mg}^{+2}$ compared to non irradiated ones under salinity. On the other hand, irradiation with gamma rays decreased lipid peroxidation, $\mathrm{Na}^{+}$and $\mathrm{Cl}^{-}$contents which may contribute in part to activate processes involved in the alleviation of the harmful effect of salt at all concentrations used $(25,50$ and $100 \mathrm{mM})$ except at the high concentration $(200 \mathrm{mM})$. Electrophoretic studies of $\alpha$-esterase, $\beta$-esterase, polyphenol oxidase, peroxidase and acid phosphatase isozymes showed wide variations in their intensities among all treatments.
\end{abstract}

Keywords: cowpea, gamma rays, isozyme, lipid peroxidation, salt stress

\section{Introduction}

Cowpea is one of the most important food legume crops in the semi-arid tropics and contains high level of protein. Cowpea is a multipurpose crop and is grown as a grain legume mainly for dry beans and green pods and also as forage, green manure and cover crop.

Salinity is one of the most important abiotic stress factors limiting plant growth and productivity (Khan and Panda, 2008; Mohamed and Gomaa, 2012). Salinity is the main environmental factor accountable for decreasing crop productivity in many geographic areas mainly in arid and semi-arid regions (Greenway and Munns, 1980). Egypt is one of the countries that suffer from severe salinity problems. For example, $33 \%$ of the cultivated land, which comprises only 3\% of total land area in Egypt, is already salinized due to low precipitation $(25 \mathrm{mM}$ annual rainfall) and irrigation with saline water (El-Hendawy $e t$ al., 2004).

There is strong evidence that salt affects photosynthetic enzymes, chlorophylls and carotenoids (Stepien and Klobus, 2006). Salinity reduces the ability of plants to utilize water and causes a reduction in growth rate, as well as changes in plant metabolic processes (Munns, 2002).

Also, salt stress and it osmotic effects have been shown to enhance the production of reactive oxygen species
(ROS) in a variety of cells resulting oxidative stress (ElBeltagi et al., 2008; Mittler, 2002). ROS are the byproducts of many degenerative reactions in crop plants, which will affect the regular metabolism by damaging the cellular components (Foyer and Noctor, 2002). Extensive study on oxidative stress has demonstrated that exposure of plants to adverse environmental conditions induces the overproduction of reactive oxygen species (ROS), such as superoxide radical $\left(\mathrm{O}_{2}^{\circ}\right), \mathrm{H}_{2} \mathrm{O}_{2}$ and hydroxyl radical (HO) in plant cells (Wise and Naylor, 1987). In addition, ROS are highly reactive to membrane lipids, protein and DNA. They are believed to be the major contributing factors to stress injuries and to cause rapid cellular damage (Afify et al., 2011; El-Beltagi, 2011; El-Beltagi et al., 2011a, Kobeasy et al., 2011; Mohamed, 2011; Mohamed et al., 2009; O'Kane et al., 1996;), particularly when plants are exposed to stress conditions such as organisms, roasting, nematode infection, micro-organisms, lead toxicity, cadmium stress, chilling stress and Fe deficiency.

Lipid peroxidation induced by free radicals, is also important in membrane deterioration (Khan and Panda, 2008). The level of lipid peroxidation, measured as malondialdehyde (MDA) content, has been considered as an indicator of salt induced oxidation in cell membranes and a tool for determining salt tolerance in plants (Hernandez and Almansa, 2002). Lipid peroxidation rate was 
increased with increase of salt stress especially in sensitive cultivars (Arora et al., 2008).

Under salt stress, plants have evolved complex mechanisms allowing for adaptation to osmotic ionic stress caused by high salinity. These mechanisms include osmotic adjustment by accumulation of compatible solutes such as proline and lowering the toxic concentration of ions in the cytoplasm by restriction of $\mathrm{Na}^{+}$influx or its sequestration into the vacuole (Binzel et al., 1988; Bohnert et al., 1999). Therefore, salt stress caused disruption of ionic equilibrium, influx of $\mathrm{Na}^{+}$, dissipates the membrane potential and facilitates the uptake of $\mathrm{Cl}^{-}$down the chemical gradient. $\mathrm{Na}^{+}$is toxic to cell metabolism and has deleterious effect on the functioning of some of the enzymes (Niu et al., 1995). High concentration of $\mathrm{Na}^{+}$causes osmotic imbalance, membrane disorganization, reduction in growth, inhibition of cell division and expansion. High $\mathrm{Na}^{+}$levels also lead to reduction in photosynthesis and production of reactive oxygen species (Yeo, 1998).

Gamma radiation can be useful for the alteration of physiological characters (Kiong et al., 2008). The biological effect of gamma rays is based on the interaction with atoms or molecules in the cell, particularly water, to produce free radicals (Kovacs and Keresztes, 2002). These radicals can damage or modify important components of plant cells and have been reported to affect differentially the morphology, anatomy, biochemistry and physiology of plants depending on the radiation dose (Ashraf et al., 2003). These effects include changes in the plant cellular structure and metabolism e.g., dilation of thylakoid membranes, alteration in photosynthesis, modulation of the antioxidative system and accumulation of phenolic compounds (Ashraf, 2009). The relatively low-doses ionizing irradiation on plants and photosynthetic microorganisms are manifested as accelerated cell proliferation, germination rate, cell growth, enzyme activity, stress resistance and crop yields (Chakravarty and Sen, 2001; El-Beltagi, 2001, 2004; El-Beltagi et al., 2011b). On the other hand, the irradiation of seeds with high doses of gamma rays disturbs the synthesis of protein, hormone balance, leaf gasexchange, water exchange and enzyme activity (Aly and El-Beltagi 2010; Hameed et al., 2008a).

The present study aims to investigate the effect of irradiation seeds with gamma rays, on improving the salt tolerance of cowpea (Vigna sinensis) plants grown under different levels of $\mathrm{NaCl}$.

\section{Materials and methods}

Plant materials, experimental design and irradiation treatments

Seeds of cowpea plants (Vigna sinensis) were obtained from the Agriculture Research Center, Ministry of Agriculture, Giza, Egypt. The seeds were thoroughly washed with continuous current of tap water for one hour. The washed seeds were sown in pots $(25 \mathrm{~cm}$ in diameter and
$25 \mathrm{~cm}$ in depth) containing $3.5 \mathrm{~kg}$ of homogeneous loamy clay soil. Ten seeds were sown in each pot. Pots were divided into four groups. The seeds of the first group did not have any treatment to serve as control, while the seeds of the second group were irrigated with $\mathrm{NaCl}$ at concentrations $(25,50,100$ and $200 \mathrm{mM})$ to raise the pots at $80 \%$ of soil water holding capacity. The seeds of the third group were irradiated with gamma rays (50 Gy) at the Middle Eastern Regional Radioisotopes center for the Arab countries (Dokki, Cairo) and the fourth group was combination between gamma rays and $\mathrm{NaCl}$. The pots were irrigated with the water holding capacity of the soil (80\%) to serve as control and the other groups with $\mathrm{NaCl}$. This experiment was conducted under natural conditions (day length $12-14$ hrs, temperature $25-27^{\otimes} \mathrm{C}$ and humidity $70 \%)$. At 60 days from sowing the plants were collected to determine the growth parameters (shoot length, root length, number of lateral roots, number of leaves, number of internodes, number of flowers, fresh and dry weights of shoots and roots and assimilating area). In addition, plants were collected and frozen in liquid nitrogen and stored in the deep freezer for further chemical analysis.

\section{Chemical analysis}

\section{Determination of photosynthetic pigments}

Chlorophyll a, Chlorophyll b and Carotenoids were determined in cowpea leaves. The spectrophotometric method recommended by Vernon and Seely (1966) was used. The pigment contents were calculated as $\mathrm{mg} / \mathrm{g}$ fresh weight of leaves.

\section{Determination of total carbohydrates}

Total carbohydrates were determined based on the method of phenol sulfuric acid as described by Dubois et al. (1956). Pure glucose was used as standard and the amount of total carbohydrates was expressed as mg glucose $/ 100 \mathrm{~g}$ dry weight.

\section{Determination of total phenols}

Levels of soluble phenols in cowpea leaves were determined in accordance with Dihazi et al. (2003). The absorbance of the developed blue colour was read at $725 \mathrm{~nm}$. Tannic acid was used as standard and the amount of soluble phenols was expressed as mg tannic acid/g dry weight.

\section{Lipid peroxidation}

Lipid peroxidation was determined by estimating the malondialdehyde content following the method of Heath and Packer (1968). The absorbance of the resulting supernatant was recorded at $532 \mathrm{~nm}$ and $600 \mathrm{~nm}$. The non-specific absorbance at $600 \mathrm{~nm}$ was subtracted from the 532 $\mathrm{nm}$ absorbance. The absorbance coefficient of malondialdehyde was calculated by using the extinction coefficient of $155 \mathrm{mM}^{-1} \mathrm{~cm}^{-1}$. 
106

\section{Determination of osmoregulators}

The proline content was estimated by the method of Bates et al. (1973). Total free amino acids (FAA) content was estimated according to Moore and Stein (1954).

\section{Determination of mineral}

$\mathrm{Na}^{+}, \mathrm{Ca}^{+2}$ and $\mathrm{Mg}^{+2}$ were determined in the acid digested of roots and shoots of cowpea plants by atomic absorption spectrometry according to A.O.A.C. (2005). N, $\mathrm{P}$ and $\mathrm{K}$ were determined according to A.O.A.C. (1995). For chloride determination, $\mathrm{Cl}^{-}$was determined by the silver ion-titration method according to Bozcuk (1970).

\section{Electrophoretic analysis of isozymes}

Isozymes $\alpha$ - and $\beta$ - esterase, acid phosphatase, peroxidase and polyphenol oxidase were analyzed on $12 \%$ polyacrylamide slab gels. Detection of esterase isozymes was carried out by the method described by Scandalios (1964), acid phosphatase isozymes was detected by method of Wendel and Weeden (1989), peroxidase was carried out by the method described by Larsen and Benson (1970) and polyphenol oxidase was done by the method Sato and Hasegawa (1976).

\section{Statistical analysis}

The data were statistically analyzed using F-test and LSD at $5 \%$ and $1 \%$ levels of probability according to SASProgramme (1982).

\section{Results and discussion}

The growth parameters (Tab. 1) of cowpea plants exhibited differential responses to the imposed salt stress and gamma irradiation. The shoot and root lengths were highly significantly increased only at the low concentra- tion of $\mathrm{NaCl}(25 \mathrm{mM})$. On the other hand all concentrations of $\mathrm{NaCl}$ significantly decreased shoot and root lengths of cowpea plants. This decrease was alleviated by gamma irradiation at all levels of $\mathrm{NaCl}$. The other growth parameters such as number of lateral roots and bacterial nodules showed highly significant increment at all treatments. Although number of leaves, leaves area, fresh weight of shoots and roots and dry weight of shoots and roots were significantly inhibited with increasing concentrations of $\mathrm{NaCl}$ expect at the low concentrations of $\mathrm{NaCl}$ ( $25 \mathrm{mM}$ ) which showed high significant increase in all the above parameters. Whereas, the irradiated seeds of cowpea plants with gamma rays under salt stress caused highly significantly increment in all the above growth parameters as compared with control $\left(\mathrm{H}_{2} \mathrm{O}\right)$ except the high concentration of $\mathrm{NaCl}(200 \mathrm{mM})$. These results are in accordance with Amirjani (2011) who found that salinity stress significantly affected plant growth components such as shoot and root lengths and fresh (FW) and dry weights (DW) of rice seedlings. Also, Nassar et al. (2004) found that, irradiating chamomile seeds with different doses of gamma rays $(0,20,40,60,80$ or $100 \mathrm{~Gy})$ before sowing increased significantly the plant height and the number of branches with increasing the dose of gamma irradiation.

As illustrated in Tab. 2, chlorophyll a and b, carotenoids and total photosynthetic pigment contents in leaves of cowpea plants were significantly increased in irradiated and non-irradiated treatment under the low concentrations of $\mathrm{NaCl}$ ( 25 and $50 \mathrm{mM}$ ). On the other hand, highly significant decrease in chlorophyll a, b, carotenoids and total photosynthetic pigment contents at the higher salinity levels (100 and $200 \mathrm{mM}$ ). Irradiating seeds with gamma rays caused enhancement in photosynthetic pigment contents under salt stress except the high concentration of $\mathrm{NaCl}(200 \mathrm{mM})$. These results are in accordance with

Tab. 1. Effect of gamma rays on growth parameters of cowpea plants under salt stress

\begin{tabular}{|c|c|c|c|c|c|c|c|c|c|c|}
\hline Treatments & $\begin{array}{l}\text { Shoot } \\
\text { length } \\
(\mathrm{cm}) \\
\end{array}$ & $\begin{array}{l}\text { Root } \\
\text { length } \\
(\mathrm{cm})\end{array}$ & $\begin{array}{c}\text { No of } \\
\text { lateral } \\
\text { roots }\end{array}$ & $\begin{array}{c}\text { No of } \\
\text { bacterial } \\
\text { nodules }\end{array}$ & $\begin{array}{l}\text { No of } \\
\text { leaves }\end{array}$ & $\begin{array}{l}\text { Area of } \\
\text { leaves } \\
\left(\mathrm{Cm}^{2}\right)\end{array}$ & $\begin{array}{c}\text { Fresh } \\
\text { weight of } \\
\text { shoot }(\mathrm{g})\end{array}$ & $\begin{array}{c}\text { Dry } \\
\text { weight of } \\
\text { shoot }(\mathrm{g})\end{array}$ & $\begin{array}{c}\text { Fresh } \\
\text { weight of } \\
\text { root }(\mathrm{g})\end{array}$ & $\begin{array}{c}\text { Dry } \\
\text { weight of } \\
\text { root }(\mathrm{g})\end{array}$ \\
\hline $0.0 \mathrm{mM} \mathrm{NaCl}$ & $16.22 \pm 1.5$ & $22.00 \pm 2.3$ & $21.06 \pm 2.1$ & $2.17 \pm 0.2$ & $4.00 \pm 0.5$ & $97.4 \pm 4.3$ & $4.17 \pm 1.0$ & $0.64 \pm 0.12$ & $1.34 \pm 0.3$ & $0.08 \pm 0.02$ \\
\hline $25 \mathrm{mM} \mathrm{NaCl}$ & $17.04 \pm 1.8^{c}$ & $24.33 \pm 2.5^{\mathrm{a}}$ & $30.2 \pm 2.6^{a}$ & $4.00 \pm 0.5^{\mathrm{a}}$ & $4.20 \pm 0.6^{a}$ & $105.3 \pm 5.8^{\mathrm{a}}$ & $4.76 \pm 0.9^{a}$ & $0.73 \pm 0.15^{a}$ & $2.01 \pm 0.5^{\mathrm{a}}$ & $0.14 \pm 0.04^{a}$ \\
\hline $50 \mathrm{mM} \mathrm{NaCl}$ & $14.46 \pm 1.3^{\mathrm{b}}$ & $20.33 \pm 2.1^{\mathrm{b}}$ & $40.8 \pm 3.0^{\mathrm{a}}$ & $2.60 \pm 0.3^{\mathrm{a}}$ & $3.80 \pm 0.4^{\mathrm{b}}$ & $94.2 \pm 4 . .0^{c}$ & $3.95 \pm 0.7^{c}$ & $0.60 \pm 0.14^{\mathrm{b}}$ & $1.18 \pm 0.2^{c}$ & $0.070 .01^{\mathrm{c}}$ \\
\hline $100 \mathrm{mM} \mathrm{NaCl}$ & $14.00 \pm 1.2^{\mathrm{b}}$ & $18.75 \pm 1.8^{\mathrm{b}}$ & $27.00 \pm 2.4^{a}$ & $2.53 \pm 0.2^{\mathrm{a}}$ & $3.80 \pm 0.4^{\mathrm{b}}$ & $81.4 \pm 3.9^{b}$ & $3.86 \pm 0.8^{b}$ & $0.57 \pm 0.16^{\mathrm{b}}$ & $0.99 \pm 0.1^{\mathrm{b}}$ & $0.06 \pm 0.00^{\mathrm{b}}$ \\
\hline $200 \mathrm{mM} \mathrm{NaCl}$ & $11.62 \pm 1.0^{\mathrm{b}}$ & $17.30 \pm 1.7^{\mathrm{b}}$ & $28.6 \pm 2.7^{\mathrm{a}}$ & $3.20 \pm 0.4^{\mathrm{a}}$ & $3.40 \pm 0.2^{b}$ & $69.4 \pm 3.5^{\mathrm{b}}$ & $3.11 \pm 0.6^{b}$ & $0.45 \pm 0.12^{b}$ & $0.62 \pm 0.1^{\mathrm{b}}$ & $0.04 \pm 0.01^{\mathrm{b}}$ \\
\hline $50 \mathrm{~Gy}$ & $18.50 \pm 1.9^{\mathrm{a}}$ & $30.88 \pm 2.7^{\mathrm{a}}$ & $24.4 \pm 2.6^{\mathrm{a}}$ & $2.60 \pm 0.2^{\mathrm{a}}$ & $4.40 \pm 0.5^{a}$ & $107.1 \pm 6.1^{\mathrm{a}}$ & $4.48 \pm 1.0^{\mathrm{a}}$ & $0.76 \pm 0.28^{a}$ & $1.61 \pm 0.4^{a}$ & $0.10 \pm 0.02^{\mathrm{a}}$ \\
\hline $\begin{array}{c}25 \mathrm{mM} \mathrm{NaCl} \\
+50 \mathrm{~Gy}\end{array}$ & $19.46 \pm 2.1^{\mathrm{a}}$ & $25.43 \pm 2.5^{a}$ & $38.1 \pm 2.9^{\mathrm{a}}$ & $4.67 \pm 0.5^{a}$ & $0.5^{\mathrm{a}}$ & $111.9 \pm 6.5^{a}$ & $5.34 \pm 1.3^{a}$ & $0.83 \pm 0.13^{\mathrm{a}}$ & $2.43 \pm 0.5^{\mathrm{a}}$ & $0.16 \pm 0.03^{a}$ \\
\hline $\begin{array}{c}50 \mathrm{mM} \mathrm{NaCl} \\
+50 \mathrm{~Gy}\end{array}$ & $19.00 \pm 2.1^{\mathrm{a}}$ & $24.50 \pm 2.2^{\mathrm{a}}$ & $49.7 \pm 3.3^{a}$ & $5.40 \pm 0.5^{\mathrm{a}}$ & $4.20 \pm 0.5$ & $108.6 \pm 6.3^{a}$ & $5.03 \pm 1.1^{\mathrm{a}}$ & $0.71 \pm 0.12^{\mathrm{a}}$ & $1.64 \pm 0.4^{a}$ & $0.11 \pm 0.01^{\mathrm{a}}$ \\
\hline $\begin{array}{c}100 \mathrm{mM} \mathrm{NaCl} \\
+50 \mathrm{~Gy}\end{array}$ & $18.33 \pm 1.8^{\mathrm{a}}$ & $23.80 \pm 2.3^{\mathrm{a}}$ & $32.3 \pm 2.8^{a}$ & $2.83 \pm 0.3^{\mathrm{a}}$ & $4.17 \pm 0.5^{a}$ & $107.2 \pm 6.0^{\mathrm{a}}$ & $4.43 \pm 0.9^{a}$ & $0.70 \pm 0.10^{\mathrm{a}}$ & $1.55 \pm 0.3^{\mathrm{a}}$ & $0.10 \pm 0.01^{\mathrm{a}}$ \\
\hline $\begin{array}{c}200 \mathrm{mM} \mathrm{NaCl} \\
+50 \mathrm{~Gy}\end{array}$ & $13.50 \pm 1.2^{\mathrm{b}}$ & $18.17 \pm 1.9^{\mathrm{b}}$ & $27.00 \pm 2.2^{\mathrm{a}}$ & $4.00 \pm 0.4^{a}$ & $3.62 \pm 0.4^{b}$ & $83.1 \pm 3.5^{\mathrm{b}}$ & $3.35 \pm 0.8^{\mathrm{b}}$ & $0.58 \pm 0.09^{\mathrm{b}}$ & $1.01 \pm 0.2^{\mathrm{b}}$ & $0.06 \pm 0.01^{\mathrm{b}}$ \\
\hline L.S.D at $1 \%$ & 0.83 & 1.27 & 2.66 & 0.33 & 0.10 & 4.40 & 0.22 & 0.03 & 0.16 & 0.011 \\
\hline
\end{tabular}

Means \pm SD $(n=10)$ of measurements on each ten plants. Means followed by a,b are highly significant increase, decrease and c significant increase and decrease different at $p \leq 0.01$, according to least significant difference (LSD) test 
Tab. 2. Effect of gamma rays on photosynthetic pigment contents in leaves of cowpea plants under salt stress

\begin{tabular}{cccccc}
\hline Treatments & $\begin{array}{c}\text { Chl a } \\
\mathrm{mg} / \mathrm{g}\end{array}$ & $\begin{array}{c}\text { Chl b } \\
\mathrm{mg} / \mathrm{g}\end{array}$ & $\begin{array}{c}\text { Chl a+b } \\
\mathrm{mg} / \mathrm{g}\end{array}$ & $\begin{array}{c}\text { Carotenoids } \\
\mathrm{mg} / \mathrm{g}\end{array}$ & $\begin{array}{c}\text { Total Pigments } \\
\mathrm{mg} / \mathrm{g}\end{array}$ \\
\hline $0.0 \mathrm{mM} \mathrm{NaCl}$ & $8.73 \pm 1.0$ & $2.83 \pm 0.3$ & $11.56 \pm 1.0$ & $4.95 \pm 0.5$ & $16.52 \pm 1.9$ \\
$25 \mathrm{mM} \mathrm{NaCl}$ & $12.53 \pm 1.5^{\mathrm{a}}$ & $4.67 \pm 0.5^{\mathrm{a}}$ & $17.20 \pm 1.5^{\mathrm{a}}$ & $6.56 \pm 0.7^{\mathrm{a}}$ & $23.76 \pm 2.5^{\mathrm{a}}$ \\
$50 \mathrm{mM} \mathrm{NaCl}$ & $10.09 \pm 1.3^{\mathrm{a}}$ & $4.39 \pm 0.5^{\mathrm{a}}$ & $14.48 \pm 1.4^{\mathrm{a}}$ & $5.55 \pm 0.6^{\mathrm{a}}$ & $20.03 \pm 1.8^{\mathrm{a}}$ \\
$100 \mathrm{mM} \mathrm{NaCl}$ & $7.78 \pm 1.0^{\mathrm{b}}$ & $2.39 \pm 0.4^{\mathrm{b}}$ & $10.17 \pm 1.0^{\mathrm{b}}$ & $4.53 \pm 0.4^{\mathrm{b}}$ & $14.70 \pm 1.2^{\mathrm{b}}$ \\
\hline $200 \mathrm{mM} \mathrm{NaCl}$ & $7.65 \pm 0.9^{\mathrm{b}}$ & $2.22 \pm 0.4^{\mathrm{b}}$ & $9.87 \pm 0.9^{\mathrm{b}}$ & $4.48 \pm 0.4^{\mathrm{b}}$ & $14.35 \pm 1.1^{\mathrm{b}}$ \\
$50 \mathrm{~Gy}$ & $11.90 \pm 1.1^{\mathrm{a}}$ & $4.66 \pm 0.6^{\mathrm{a}}$ & $16.56 \pm 1.4^{\mathrm{a}}$ & $6.49 \pm 0.7^{\mathrm{a}}$ & $23.05 \pm 1.9^{\mathrm{a}}$ \\
$25 \mathrm{mM} \mathrm{NaCl}+50 \mathrm{~Gy}$ & $15.13 \pm 1.6^{\mathrm{a}}$ & $5.25 \pm 0.7^{\mathrm{a}}$ & $20.38 \pm 1.7^{\mathrm{a}}$ & $8.89 \pm 0.8^{\mathrm{a}}$ & $29.27 \pm 2.4^{\mathrm{a}}$ \\
$50 \mathrm{mM} \mathrm{NaCl}+50 \mathrm{~Gy}$ & $13.30 \pm 1.4^{\mathrm{a}}$ & $4.99 \pm 0.5^{\mathrm{a}}$ & $18.29 \pm 1.6^{\mathrm{a}}$ & $7.27 \pm 0.7^{\mathrm{a}}$ & $25.56 \pm 2.1^{\mathrm{a}}$ \\
$100 \mathrm{mM} \mathrm{NaCl}+50 \mathrm{~Gy}$ & $10.37 \pm 1.1^{\mathrm{a}}$ & $3.69 \pm 0.4^{\mathrm{a}}$ & $14.06 \pm 1.5^{\mathrm{a}}$ & $6.25 \pm 0.6^{\mathrm{a}}$ & $20.30 \pm 2.0^{\mathrm{a}}$ \\
$200 \mathrm{mM} \mathrm{NaCl}+50 \mathrm{~Gy}$ & $8.22 \pm 0.8^{\mathrm{d}}$ & $2.46 \pm 0.3^{\mathrm{b}}$ & $10.68 \pm 1.2^{\mathrm{c}}$ & $4.74 \pm 0.5^{\mathrm{d}}$ & $15.42 \pm 1.3^{\mathrm{c}}$ \\
\hline L.S.D at $1 \%$ & 0.79 & 0.36 & 1.14 & 0.44 & 1.57 \\
\hline
\end{tabular}

Means \pm SD $(n=3)$ of measurements on each three plants. Means followed by a,b are highly significant increase, decrease, c significant increase and decrease and d non significant effect different at $p \leq 0.01$, according to least significant difference (LSD) test

the results of Dhanapackiam and Muhammad (2010) who found that the levels of salinization $(40$ and $50 \mathrm{mM} \mathrm{NaCl}$ ) induced a significant decrease in the contents of pigment fractions (chlorophyll a and b) and consequently of the total photosynthetic pigment content as compared with control plants. While, the total photosynthetic pigment content of the leaves of Sesbania grandiflora seedlings exhibited a little increase when grown at 10 and $20 \mathrm{mM}$ $\mathrm{NaCl}$. In addition, an increase in chlorophyll $a, b$ and total photosynthetic pigment levels was observed in Paulownia tomentosa plants that were exposed to gamma irradiation as compared to the non-irradiated plants (Alikamanoglu et al., 2007). Modulation in photosynthesis in irradiated plants might partly contribute to increased growth (Wi et al., 2007).

The results in Tab. (3) showed that the total carbohydrate content in shoots and roots of cowpea plants was highly significantly increased at the low concentrations of $\mathrm{NaCl}(25$ and $50 \mathrm{mM})$. On the other hand, the high concentrations of $\mathrm{NaCl}$ (100 and $200 \mathrm{mM}$ ) caused highly significantly decreases in the same contents as compared with control plants. These results are in accordance with those of Almodares et al. (2008) who reported that the amount of soluble carbohydrate decreases in two sweet sorghum cultivars (Keller and Sofra) as salinity increased. Seeds irradiated with gamma rays showed highly significantly increases in total carbohydrate contents in shoots and roots of cowpea plants under all salt stress as compared with the corresponding controls. These results are in accordance with Moussa (2011) who found that gamma irradiation (20 Gy) increased the soluble sugars in drought stressed soybean leaves.

Also, the results presented in Tab. 3 revealed that the total phenol level was significantly increased in root and shoot of cowpea plants grown under all $\mathrm{NaCl}$ levels alone or in combination with gamma rays as compared with those of untreated and treated plants. Similarly, Ashraf et al. (2010) found that, the phenolic contents of bread wheat (Triticum aestivum L.) were affected significantly due to salt stress $(150 \mathrm{mM})$. Also, Aly (2010) reported that $\gamma$-irradiation increased the biosynthesis of phenolic

Tab. 3. Effect of gamma rays on total carbohydrates, total phenols contents and lipid peroxidation in cowpea plants under salt stress

\begin{tabular}{|c|c|c|c|c|c|c|}
\hline \multirow[t]{2}{*}{ Treatments } & \multicolumn{2}{|c|}{$\begin{array}{c}\text { Total carbohydrates } \\
\mathrm{mg} / 100 \mathrm{~g}\end{array}$} & \multicolumn{2}{|c|}{$\begin{array}{c}\text { Total phenol } \\
\mu \mathrm{g} / \mathrm{g}\end{array}$} & \multicolumn{2}{|c|}{$\begin{array}{l}\text { Lipid peroxidation } \\
\text { n mole/g F.wt }\end{array}$} \\
\hline & Root & Shoot & Root & Shoot & Root & Shoot \\
\hline $0.0 \mathrm{mM} \mathrm{NaCl}$ & $32.1 \pm 2.5$ & $41.2 \pm 3.0$ & $7.7 \pm 0.9$ & $70.0 \pm 2.5$ & $5.1 \pm 0.5$ & $9.3 \pm 0.4$ \\
\hline $25 \mathrm{mM} \mathrm{NaCl}$ & $44.3 \pm 3.3^{\mathrm{a}}$ & $47.6 \pm 3.3^{\mathrm{a}}$ & $31.6 \pm 1.9^{a}$ & $136.3 \pm 3.7^{\mathrm{a}}$ & $12.9 \pm 0.9^{\mathrm{a}}$ & $13.8 \pm 0.6^{\mathrm{a}}$ \\
\hline $50 \mathrm{mM} \mathrm{NaCl}$ & $40.7 \pm 2.7^{\mathrm{a}}$ & $44.4 \pm 2.9^{a}$ & $25.3 \pm 1.5^{a}$ & $98.2 \pm 2.6^{\mathrm{a}}$ & $15.4 \pm 1.1^{\mathrm{a}}$ & $20.0 \pm 0.9^{a}$ \\
\hline $100 \mathrm{mM} \mathrm{NaCl}$ & $30.1 \pm 2.2^{b}$ & $37.4 \pm 2.4^{b}$ & $24.0 \pm 1.4^{\mathrm{a}}$ & $79.3 \pm 2.1^{\mathrm{a}}$ & $18.7 \pm 1.3^{\mathrm{a}}$ & $23.3 \pm 1.0^{\mathrm{a}}$ \\
\hline $200 \mathrm{mM} \mathrm{NaCl}$ & $29.4 \pm 2.0^{\mathrm{b}}$ & $34.4 \pm 2.2^{b}$ & $10.9 \pm 1.0^{\mathrm{a}}$ & $75.0 \pm 2.0^{\mathrm{a}}$ & $21.5 \pm 1.5^{\mathrm{a}}$ & $35.7 \pm 1.2^{\mathrm{a}}$ \\
\hline $50 \mathrm{~Gy}$ & $38.4 \pm 2.4^{a}$ & $45.4 \pm 2.8^{\mathrm{a}}$ & $32.0 \pm 1.8^{\mathrm{a}}$ & $114.6 \pm 2.8^{a}$ & $8.3 \pm 0.8^{\mathrm{a}}$ & $12.7 \pm 0.5^{a}$ \\
\hline $25 \mathrm{mM} \mathrm{NaCl} 50$ Gy & $46.6 \pm 3.5^{a}$ & $49.3 \pm 3.1^{\mathrm{a}}$ & $34.3 \pm 2.0^{\mathrm{a}}$ & $155.8 \pm 4.0^{\mathrm{a}}$ & $9.4 \pm 0.9^{\mathrm{a}}$ & $12.9 \pm 0.6^{\mathrm{a}}$ \\
\hline $50 \mathrm{mM} \mathrm{NaCl}+50 \mathrm{~Gy}$ & $42.2 \pm 2.9^{\mathrm{a}}$ & $46.3 \pm 3.5^{a}$ & $33.3 \pm 2.1^{\mathrm{a}}$ & $98.8 \pm 1.9^{a}$ & $11.6 \pm 0.8^{\mathrm{a}}$ & $13.7 \pm 0.6^{\mathrm{a}}$ \\
\hline $100 \mathrm{mM} \mathrm{NaCl}+50 \mathrm{~Gy}$ & $37.2 \pm 2.4^{\mathrm{a}}$ & $45.1 \pm 3.2^{\mathrm{a}}$ & $32.3 \pm 2.0^{\mathrm{a}}$ & $83.7 \pm 1.7^{\mathrm{a}}$ & $12.4 \pm 0.9^{a}$ & $15.1 \pm 0.7^{\mathrm{a}}$ \\
\hline $200 \mathrm{mM} \mathrm{NaCl}+50 \mathrm{~Gy}$ & $30.9 \pm 2.3^{d}$ & $37.4 \pm 2.6^{b}$ & $21.2 \pm 1.6^{a}$ & $80.3 \pm 1.5^{a}$ & $12.9 \pm 0.4^{\mathrm{a}}$ & $15.6 \pm 0.7^{a}$ \\
\hline L.S.D at $1 \%$ & 1.93 & 1.54 & 2.93 & 8.75 & 1.49 & 2.43 \\
\hline
\end{tabular}

Means \pm SD $(n=3)$ of measurements on each three plants. Means followed by a and b are highly significant increase and decrease different at $p \leq 0.01$, according to least significant difference (LSD) test 
108

compounds in Culantro (Eryngium foetidum L.) fresh plantlets.

Tab. 3 showed that all $\mathrm{NaCl}$ levels induced intense lipid peroxidation as indicated by the increase in the thiobarbituric acid reactive substances (TBARs) product in root and shoot of cowpea plants. Treatment with gamma irradiation either alone or in combination with different $\mathrm{NaCl}$ levels reduced the level of TBARs. The increase in the MDA content at all salinity levels may be due to oxidative damage affecting both organelles (chloroplasts and mitochondria). These results are in accordance with Moussa (2011) who found that gamma irradiation decreased the malondialdehyde concentration of the leaves of soybean plants under drought stress. Also, Hameed et al. (2008b) reported that lipid peroxidation, which have an important role in abiotic stresses decreased significantly from 100200 Gy radiation dose in chickpea. Similarly, Baek et al. (2006) found that gamma irradiation lowered the MDA content in rice plants under salt stress.

Salt stress significantly increased proline and total free amino acid contents of roots and shoots of cowpea plants as compared to that of control (Tab. 4). Treatment with gamma irradiation significantly induced additional increases in the same contents as compared to the value of the control under salt stress. These results are in accordance with Mahajan and Tuteja (2005) who found that proline accumulation may contribute to the adjustment at the cellular level which may acts as an enzyme protectant and stabilizing the structure of macro molecules. Proline also acts as a major reservoir of energy and nitrogen for utilization upon exposure to salinity. It was suggested that proline accumulation may be caused by increased proteolysis or by decreased protein synthesis.

The higher concentration of proline under salt stress is favorable to plants as proline participates in the osmotic potential of leaf and thus in the osmotic adjustment. Also, proline can confer enzyme protection and increase membrane stability under various condition. Proline ac-
Tab. 4. Effect of gamma rays on proline and total free amino acids contents in cowpea plants under salt stress

\begin{tabular}{ccccc}
\hline \multirow{2}{*}{ Treatments } & \multicolumn{2}{c}{ Proline } & \multicolumn{2}{c}{ Total free amino acid } \\
& \multicolumn{2}{c}{$\mu \mathrm{g} / \mathrm{g}$} & \multicolumn{2}{c}{$\mu \mathrm{g}$} \\
\cline { 2 - 5 } & Root & Shoot & Root & Shoot \\
\hline $0.0 \mathrm{mM} \mathrm{NaCl}$ & $2.88 \pm 0.2$ & $3.04 \pm 0.2$ & $23.0 \pm 1.5$ & $26.2 \pm 2.0$ \\
$25 \mathrm{mM} \mathrm{NaCl}$ & $3.78 \pm 0.3^{\mathrm{a}}$ & $3.54 \pm 0.2^{\mathrm{a}}$ & $30.2 \pm 2.4^{\mathrm{a}}$ & $31.9 \pm 2.4^{\mathrm{a}}$ \\
$50 \mathrm{mM} \mathrm{NaCl}$ & $4.25 \pm 0.4^{\mathrm{a}}$ & $3.96 \pm 0.3^{\mathrm{a}}$ & $34.0 \pm 2.5^{\mathrm{a}}$ & $36.7 \pm 2.6^{\mathrm{a}}$ \\
$100 \mathrm{mM} \mathrm{NaCl}$ & $4.38 \pm 0.5^{\mathrm{a}}$ & $4.03 \pm 0.4^{\mathrm{a}}$ & $35.0 \pm 2.6^{\mathrm{a}}$ & $38.8 \pm 2.5^{\mathrm{a}}$ \\
$200 \mathrm{mM} \mathrm{NaCl}$ & $5.20 \pm 0.5^{\mathrm{a}}$ & $6.11 \pm 0.5^{\mathrm{a}}$ & $41.6 \pm 2.9^{\mathrm{a}}$ & $46.5 \pm 2.9^{\mathrm{a}}$ \\
$50 \mathrm{~Gy}$ & $3.48 \pm 0.2^{\mathrm{a}}$ & $3.20 \pm 0.3^{\mathrm{a}}$ & $27.8 \pm 1.7^{\mathrm{a}}$ & $55.9 \pm 3.3^{\mathrm{a}}$ \\
$25 \mathrm{mM} \mathrm{NaCl}+50$ Gy & $4.29 \pm 0.3^{\mathrm{a}}$ & $3.93 \pm 0.3^{\mathrm{a}}$ & $34.3 \pm 2.0^{\mathrm{a}}$ & $59.5 \pm 3.5^{\mathrm{a}}$ \\
$50 \mathrm{mM} \mathrm{NaCl}+50$ Gy & $4.44 \pm 0.3^{\mathrm{a}}$ & $4.02 \pm 0.4^{\mathrm{a}}$ & $35.5 \pm 2.2^{\mathrm{a}}$ & $69.0 \pm 3.9^{\mathrm{a}}$ \\
$100 \mathrm{mM} \mathrm{NaCl}+50$ Gy & $5.03 \pm 0.4^{\mathrm{a}}$ & $4.59 \pm 0.4^{\mathrm{a}}$ & $40.2 \pm 2.4^{\mathrm{a}}$ & $82.6 \pm 4.0^{\mathrm{a}}$ \\
$200 \mathrm{mM} \mathrm{NaCl}+50$ Gy & $5.46 \pm 0.5^{\mathrm{a}}$ & $6.36 \pm 0.5^{\mathrm{a}}$ & $43.7 \pm 2.6^{\mathrm{a}}$ & $107.5 \pm 4.5^{\mathrm{a}}$ \\
L.S.D at $1 \%$ & 0.25 & 0.35 & 1.96 & 7.80 \\
\hline
\end{tabular}

Means \pm SD $(n=3)$ of measurements on each three plants. Means followed by a is highly significant increase different at $p \leq 0.01$, according to least significant difference (LSD) test

cumulation may also help in nonenzymic free radical detoxifications (Khan et al., 2002). Moreover, free amino acids accumulation in plants under salt stress has been attributed to alteration in biosynthesis and degradation processes of amino acids and proteins (Silva et al., 2008). The increase in amino acids may be attributed to the changes caused by water deficit which promote protein degradation by protease. There was significant increase in the level of amino acids in Vigna unguiculata L. plants under water stress (Lobato et al., 2008).

High doses of gamma radiation were reported to induce oxidative stress. To avoid oxidative damage, plants have evolved various protective mechanisms to counteract the effects of reactive oxygen species in cellular compartments. This defense was brought by alteration in the pattern of gene expression. This led to modulation of certain metabolic and defensive pathways. One of the protective mechanisms in the synthesis of osmolytes which is essenTab. 5a. Effect of gamma rays on (N, P, K) contents in cowpea plants under salt stress

\begin{tabular}{|c|c|c|c|c|c|c|}
\hline \multirow[t]{2}{*}{ Treatments } & \multicolumn{2}{|c|}{$\begin{array}{c}\mathrm{N} \\
\mathrm{g} / 100 \mathrm{~g}\end{array}$} & \multicolumn{2}{|c|}{$\begin{array}{c}\mathrm{P} \\
\mathrm{g} / 100 \mathrm{~g}\end{array}$} & \multicolumn{2}{|c|}{$\begin{array}{c}\mathrm{K} \\
\mathrm{g} / 100 \mathrm{~g}\end{array}$} \\
\hline & Root & Shoot & Root & Shoot & Root & Shoot \\
\hline $0.0 \mathrm{mM} \mathrm{NaCl}$ & 2.51 & 2.90 & 0.131 & 0.159 & 1.55 & 1.41 \\
\hline $25 \mathrm{mM} \mathrm{NaCl}$ & $2.22^{\mathrm{b}}$ & $2.70^{\mathrm{b}}$ & $0.125^{\mathrm{d}}$ & $0.143^{\mathrm{b}}$ & $1.44^{\mathrm{b}}$ & $1.19^{\mathrm{b}}$ \\
\hline $50 \mathrm{mM} \mathrm{NaCl}$ & $2.12^{\mathrm{b}}$ & $2.51^{\mathrm{b}}$ & $0.105^{\mathrm{b}}$ & $0.120^{\mathrm{b}}$ & $1.40^{\mathrm{b}}$ & $0.80^{\mathrm{b}}$ \\
\hline $100 \mathrm{mM} \mathrm{NaCl}$ & $1.93^{\mathrm{b}}$ & $2.32^{\mathrm{b}}$ & $0.097^{\mathrm{b}}$ & $0.113^{\mathrm{b}}$ & $1.38^{\mathrm{b}}$ & $0.78^{b}$ \\
\hline $200 \mathrm{mM} \mathrm{NaCl}$ & $1.74^{\mathrm{b}}$ & $2.12^{\mathrm{b}}$ & $0.064^{\mathrm{b}}$ & $0.112^{\mathrm{b}}$ & $1.29^{\mathrm{b}}$ & $0.72^{\mathrm{b}}$ \\
\hline $50 \mathrm{~Gy}$ & $2.62^{c}$ & $3.01^{\mathrm{d}}$ & $0.238^{\mathrm{b}}$ & $0.262^{\mathrm{a}}$ & $1.87^{\mathrm{a}}$ & $1.51^{\mathrm{c}}$ \\
\hline $25 \mathrm{mM} \mathrm{NaCl}+50 \mathrm{~Gy}$ & $2.80^{\mathrm{a}}$ & $4.00^{\mathrm{a}}$ & $0.187^{a}$ & $0.207^{\mathrm{a}}$ & $1.73^{\mathrm{a}}$ & $1.72^{\mathrm{a}}$ \\
\hline $50 \mathrm{mM} \mathrm{NaCl}+50 \mathrm{~Gy}$ & $2.72^{\mathrm{a}}$ & $3.5^{\mathrm{a}}$ & $0.166^{\mathrm{a}}$ & $0.186^{\mathrm{a}}$ & $1.67^{\mathrm{a}}$ & $1.65^{\mathrm{a}}$ \\
\hline $100 \mathrm{mM} \mathrm{NaCl}+50 \mathrm{~Gy}$ & $2.66^{a}$ & $3.42^{\mathrm{a}}$ & $0.141^{\mathrm{d}}$ & $0.179^{a}$ & $1.59^{c}$ & $1.60^{\mathrm{a}}$ \\
\hline $200 \mathrm{mM} \mathrm{NaCl}+50 \mathrm{~Gy}$ & $1.90^{\mathrm{b}}$ & $2.55^{\mathrm{b}}$ & $0.112^{b}$ & $0.140^{\mathrm{b}}$ & $1.40^{\mathrm{b}}$ & $1.13^{\mathrm{b}}$ \\
\hline L.S.D at $1 \%$ & 0.119 & 0.181 & 0.015 & 0.015 & 0.056 & 0.118 \\
\hline
\end{tabular}

Means followed by a,b are highly significant increase, decrease and c significant increase and decrease different at $p \leq 0.01$, according to least significant difference (LSD) test 
Tab. 5b. Effect of gamma rays on $(\mathrm{Na}, \mathrm{Cl}, \mathrm{Ca}, \mathrm{Mg})$ contents in cowpea plants under salt stress

\begin{tabular}{|c|c|c|c|c|c|c|c|c|}
\hline \multirow[t]{2}{*}{ Treatments } & \multicolumn{2}{|c|}{$\begin{array}{c}\mathrm{Na} \\
\mathrm{g} / 100 \mathrm{~g}\end{array}$} & \multicolumn{2}{|c|}{$\begin{array}{c}\mathrm{Cl} \\
\mathrm{g} / 100 \mathrm{~g}\end{array}$} & \multicolumn{2}{|c|}{$\begin{array}{c}\mathrm{Ca} \\
\mathrm{g} / 100 \mathrm{~g}\end{array}$} & \multicolumn{2}{|c|}{$\begin{array}{c}\mathrm{Mg} \\
\mathrm{g} / 100 \mathrm{~g}\end{array}$} \\
\hline & Root & Shoot & Root & Shoot & Root & Shoot & Root & Shoot \\
\hline $0.0 \mathrm{mM} \mathrm{NaCl}$ & 0.057 & 0.137 & 0.62 & 0.37 & 0.431 & 0.662 & 0.317 & 0.374 \\
\hline $25 \mathrm{mM} \mathrm{NaCl}$ & $0.069^{\mathrm{a}}$ & $0.171^{\mathrm{a}}$ & $0.87^{a}$ & $0.44^{c}$ & $0.397^{c}$ & $0.616^{\mathrm{b}}$ & $0.307^{\mathrm{d}}$ & $0.351^{\mathrm{c}}$ \\
\hline $50 \mathrm{mM} \mathrm{NaCl}$ & $0.080^{\mathrm{a}}$ & $0.205^{\mathrm{a}}$ & $0.94^{\mathrm{a}}$ & $0.49^{\mathrm{a}}$ & $0.384^{\mathrm{b}}$ & $0.593^{\mathrm{b}}$ & $0.275^{\mathrm{b}}$ & $0.317^{\mathrm{b}}$ \\
\hline $100 \mathrm{mM} \mathrm{NaCl}$ & $0.092^{\mathrm{a}}$ & $0.217^{\mathrm{a}}$ & $1.08^{\mathrm{a}}$ & $0.54^{a}$ & $0.291^{b}$ & $0.532^{\mathrm{b}}$ & $0.263^{b}$ & $0.281^{b}$ \\
\hline $200 \mathrm{mM} \mathrm{NaCl}$ & $0.103^{a}$ & $0.228^{a}$ & $1.15^{\mathrm{a}}$ & $1.01^{\mathrm{a}}$ & $0.268^{b}$ & $0.461^{\mathrm{b}}$ & $0.258^{b}$ & $0.265^{b}$ \\
\hline $50 \mathrm{~Gy}$ & $0.046^{\mathrm{b}}$ & $0.135^{\mathrm{d}}$ & $0.51^{\mathrm{b}}$ & $0.23^{\mathrm{b}}$ & $0.593^{\mathrm{a}}$ & $0.686^{c}$ & $0.333^{c}$ & $0.412^{\mathrm{a}}$ \\
\hline $25 \mathrm{mM} \mathrm{NaCl}+50 \mathrm{~Gy}$ & $0.045^{b}$ & $0.127^{c}$ & $0.54^{\mathrm{b}}$ & $0.20^{\mathrm{b}}$ & $0.523^{\mathrm{a}}$ & $0.686^{c}$ & $0.338^{a}$ & $0.392^{\mathrm{c}}$ \\
\hline $50 \mathrm{mM} \mathrm{NaCl}+50 \mathrm{~Gy}$ & $0.048^{b}$ & $0.130^{\mathrm{d}}$ & $0.56^{c}$ & $0.22^{b}$ & $0.577^{\mathrm{a}}$ & $0.693^{\mathrm{a}}$ & $0.363^{a}$ & $0.458^{a}$ \\
\hline $100 \mathrm{mM} \mathrm{NaCl}+50$ Gy & $0.050^{\mathrm{b}}$ & $0.132^{\mathrm{d}}$ & $0.58^{\mathrm{d}}$ & $0.24^{\mathrm{b}}$ & $0.602^{a}$ & $0.751^{a}$ & $0.482^{a}$ & $0.529^{a}$ \\
\hline $200 \mathrm{mM} \mathrm{NaCl}+50 \mathrm{~Gy}$ & $0.052^{c}$ & $0.135^{\mathrm{d}}$ & $0.60^{\mathrm{d}}$ & $0.27^{\mathrm{b}}$ & $0.315^{b}$ & $0.514^{\mathrm{b}}$ & $0.309^{d}$ & $0.284^{\mathrm{b}}$ \\
\hline L.S.D at $1 \%$ & 0.006 & 0.012 & 0.074 & 0.075 & 0.039 & 0.028 & 0.019 & 0.026 \\
\hline
\end{tabular}

Means followed by a,b are highly significant increase, decrease and c significant increase and decrease different at $p \leq 0.01$, according to least significant difference (LSD) test

tial to plant growth was proline synthesis (Esfandiari et al., 2008).

It is clear from the results in Tab. 5 that salinity caused highly significantly increased in the $\mathrm{Na}^{+}$and $\mathrm{Cl}^{-}$content and decrease in the contents of $\mathrm{N}, \mathrm{P}, \mathrm{K}^{+}, \mathrm{Mg}^{+2}$ and $\mathrm{Ca}^{+2}$ in the roots and shoots of cowpea plants. It is probably that salt stress causes nutrient deficiency due to competition between $\mathrm{Na}^{+}$and other nutrients such as $\mathrm{K}^{+}, \mathrm{Mg}^{+2}$ and $\mathrm{Ca}^{+2}$. Other possibility is that reduction in uptake of mineral nutrients under saline conditions may occur due to $\mathrm{Na}$ induced blockage or reduced activity of the transporters, resulting in ionic imbalance of $\mathrm{K}, \mathrm{Ca}^{+2}$ and $\mathrm{Mg}^{+2}$ as compared to $\mathrm{Na}^{+}$. After treating with gamma irradiation, reduction in the $\mathrm{Na}^{+}$and $\mathrm{Cl}^{-}$uptake of plants and/or increased the $\mathrm{K}^{+}, \mathrm{N}, \mathrm{P}, \mathrm{Mg}^{+2}$ and $\mathrm{Ca}^{+2}$ uptakes compared to control treatment under salt stress. These results are in accordance with Shereen et al. (2009) who found that shoot ions concentration showed an increase in accumulation of $\mathrm{Na}^{+}$coupled with decrease in $\mathrm{K}^{+}$concentration in rice plants under salt stress.

The present study showed that, the radiation dose of 50 Gy was found to be very effective for improving the ionic balance in roots and shoots of cowpea plants. The reduction in the uptake of toxic ion like $\mathrm{Na}^{+}$may have exerted positive role in enhancement of cellular functions like pigment production and other growth attributes, which collectively resulted in better growth of cowpea plants under saline conditions.

Reduction in plant growth due to salinity is a common feature as was observed in cowpea plants. This reduction in growth may be attributed to osmotic effects which the water availability to plants is hindered. There may be salt specific effects. If absorption of excess quantity of toxic ions takes place, the concentration inside the plant may reach to toxic level and causes ionic imbalances at cellular and organ level. These imbalances may cause hindrances in various physiological functions and ultimately the growth and yield is affected (Hameed et al., 2008a). One of the most important factors under saline environment may be the overall control mechanism of salt uptake through root and its subsequent distribution to shoot. The other factor is the maintenance of mineral nutrients such as $\mathrm{K}^{+}$ and $\mathrm{Ca}^{+2}$ which are essentially required for the activities of enzymes, proteins synthesis and integrity of cell wall and plasma membrane (Taiz and Zeiger, 2006).

The electrophoretic profiles of five enzymes; $\alpha$ and $\beta$ esterase, polyphenol oxidase, peroxidase and acid phosphatase of cowpea leaves under different concentrations of $\mathrm{NaCl}$ alone or in combination of gamma ray are presented in Tab. 6 and Fig. 1.

$\alpha$ - esterase electrophoretic patterns are illustrated in Tab. 6 and Fig 1. Four bands were exhibited with different intensities among all treatments. Band No. 1 and 4 which have $R_{f} 0.026$ and 0.95 were present in all treatments (common bands). The other two bands were present in some treatments and absent in the others (polymorphic). $\mathrm{NaCl}$ concentrations $(25,50,100$ and $200 \mathrm{mM}$ ) increased the activity of esterase isozymes. The activity of esterase increased at concentrations of $\mathrm{NaCl}(25,100$ and 200 $\mathrm{mM}$ ) after treatment with gamma rays. These results are in agreement with Hassanein (1999), who found that salinity increase esterase isozymes and the highest numbers of esterase isozymes were detected under the highest $\mathrm{NaCl}$ concentration. Also, El-Sayed et al. (2007) who found that salinity and gamma rays caused the appearance and disappearance of bands in two wheat cultivars.

In Tab. 6, two bands with different intensities were observed among $\beta$ - esterase profiles of all treatments. One band was presented in all treatments (monomorphic bands) at $R_{f} 0.20$. The other band was presented in some treatments and absent in the others. The band which has $\mathrm{R}_{\mathrm{f}} 0.59$ become very intensified at concentrations of $\mathrm{NaCl}$ $(25,100$ and $200 \mathrm{mM})$ after treatment with gamma rays as compared with control treatment. These results are similar to Mohamed (2005) who found that under salt stress, 150 
Tab. 6. Effect of gamma rays on different isozymes profiles in cowpea leaves under salt stress

\begin{tabular}{|c|c|c|c|c|c|c|c|c|c|c|c|}
\hline \multirow[b]{2}{*}{ Enzymes } & \multirow[b]{2}{*}{$\mathrm{R}_{\mathrm{f}}$} & \multicolumn{10}{|c|}{ Treatments } \\
\hline & & Control & $50 \mathrm{~Gy}$ & $\begin{array}{c}25 \mathrm{mM} \\
\mathrm{NaCl}\end{array}$ & $\begin{array}{c}25 \mathrm{mM} \\
\mathrm{NaCl}+50 \\
\mathrm{~Gy}\end{array}$ & $\begin{array}{c}50 \mathrm{mM} \\
\mathrm{NaCl}\end{array}$ & $\begin{array}{c}50 \mathrm{mM} \\
\mathrm{NaCl}+50 \\
\mathrm{~Gy}\end{array}$ & $\begin{array}{c}100 \mathrm{mM} \\
\mathrm{NaCl}\end{array}$ & $\begin{array}{c}100 \mathrm{mM} \\
\mathrm{NaCl}+50 \\
\mathrm{~Gy}\end{array}$ & $\begin{array}{c}200 \mathrm{mM} \\
\mathrm{NaCl}\end{array}$ & $\begin{array}{c}200 \mathrm{mM} \\
\mathrm{NaCl}+50 \\
\mathrm{~Gy}\end{array}$ \\
\hline \multirow{4}{*}{$\alpha$-esterase } & 0.026 & 1.21 & 1.93 & 3.42 & 3.99 & 3.17 & 2.9 & 2 & 2.41 & 2.88 & 3.16 \\
\hline & 0.57 & - & - & - & 8.38 & 6.42 & 11.7 & 6.89 & 9.09 & 7.06 & 5.43 \\
\hline & 0.68 & 16.6 & - & 11.6 & 14.7 & 12.2 & 10.6 & 18.8 & 14 & 10.5 & 14.3 \\
\hline & 0.95 & 9.77 & 11.8 & 8.54 & 6.69 & 7.8 & 5.66 & 6.56 & 5.04 & 11.5 & 10.3 \\
\hline \multicolumn{2}{|c|}{ Total No. of bands } & 3 & 2 & 3 & 4 & 4 & 4 & 4 & 4 & 4 & 4 \\
\hline \multirow{2}{*}{$\beta$-esterase } & 0.20 & 4.07 & 4.31 & 5.82 & 5.69 & 4.9 & 4.82 & 4.71 & 6.07 & 5.21 & 4.69 \\
\hline & 0.59 & 11.7 & - & - & 12 & 4.59 & 5.98 & 9.54 & 13.1 & 5.86 & 13.3 \\
\hline \multicolumn{2}{|c|}{ Total No. of bands } & 2 & 1 & 1 & 2 & 2 & 2 & 2 & 2 & 2 & 2 \\
\hline \multirow{10}{*}{$\begin{array}{l}\text { Polyphenol } \\
\text { oxidase }\end{array}$} & 0.03 & - & - & 7.23 & 8.05 & 4.44 & 5.77 & 4.93 & 5.49 & 4.73 & 6.56 \\
\hline & 0.09 & 5 & 6.29 & 6.88 & 8.33 & 5.23 & 4.91 & - & - & - & - \\
\hline & 0.15 & 6.98 & 7.7 & 6.61 & 6.21 & 6.95 & 7.32 & 5.19 & 6.07 & 7.28 & 6.93 \\
\hline & 0.24 & 6.95 & 7.18 & 8.18 & 7.75 & 5.86 & 5.87 & 7.4 & 4.98 & 6.56 & 4.88 \\
\hline & 0.34 & 8.52 & 7 & 7.9 & 7.47 & - & - & - & - & - & - \\
\hline & 0.40 & - & - & 10.6 & 7.33 & 11.1 & 11.6 & 11.5 & 10.6 & 9.38 & 14.9 \\
\hline & 0.58 & 18.3 & 6.43 & 2.73 & - & - & - & - & - & 5.53 & - \\
\hline & 0.67 & - & 3.78 & 2.56 & 3.12 & 6.33 & 2.47 & 6.59 & 4.33 & 7.91 & 5.74 \\
\hline & 0.72 & - & - & - & 2 & 4.04 & 3.1 & 3.36 & 4.52 & - & - \\
\hline & 0.87 & - & - & - & - & 4.27 & 4.08 & 5.01 & 5.14 & 5.48 & 4.83 \\
\hline \multicolumn{2}{|c|}{ Total No. of bands } & 5 & 5 & 8 & 8 & 8 & 8 & 7 & 7 & 7 & 6 \\
\hline peroxidase & 0.038 & 4.04 & 4.65 & 4.23 & 3.88 & 4.56 & 5.47 & 5.77 & 8.14 & 9.89 & 9.32 \\
\hline \multicolumn{2}{|c|}{ Total No. of bands } & 1 & 1 & 1 & 1 & 1 & 1 & 1 & 1 & 1 & 1 \\
\hline Acid phosphatase & 0.044 & 12.7 & 17.4 & 25.3 & 22.5 & 15.1 & 14.0 & 13.5 & 11.9 & 13.5 & 12.1 \\
\hline \multicolumn{2}{|c|}{ Total No. of bands } & 1 & 1 & 1 & 1 & 1 & 1 & 1 & 1 & 1 & 1 \\
\hline
\end{tabular}

$\mathrm{mM} \mathrm{NaCl}$ caused enhancement of the esterase isozyme bands in shoots and roots of maize plants.

Polyphenol oxidase electrophoretic patterns are illustrated in Tab. 6 and Fig. 1. Eight bands with different intensities were observed among the profiles of all treatments. Two bands were presented in all treatments (monomorphic bands) at $\mathrm{R}_{f} 0.15$ and 0.24 . The other bands were presented in some treatments and absent in the others (polymorphic bands). These results are in agreement with El-Sayed et al. (2007) who found that salinity and gamma rays caused the appearance and disappearance of bands in two wheat cultivars.

Expression of the peroxidase isozyme was detected in cowpea leaves treated with different concentrations of $\mathrm{NaCl}$ alone or in combination with gamma rays. The results in Tab. 6 and Fig. 1 showed that one band was exhibited at $R_{f} 0.038$ with different intensities in untreated and salt treated plants. The activity of this band increased with increasing $\mathrm{NaCl}$ concentrations alone or in irradiated plants. These results indicated that salt stress increased the accumulation of the peroxidase enzyme and that the encoding gene ( $\mathrm{s}$ ) was accelerated in response to salt stress. These results are in harmony with the findings of El- Baz et al. (2003), who used peroxidase isozyme as marker for salt stress tolerance in cucumber plants. This behavior may be due to its ability to tolerate salt stress or due to the effect of salt stress which may cause some shift in gene expression. In addition, The results from the present study are in conformity with the study by Rashid et al. (2001) on the detection of genetic variation using molecular techniques among the irradiated and salt stress $(200 \mathrm{mM} \mathrm{NaCl})$ calli.

An isozyme profile of acid phosphatase in cowpea leaves grown under $\mathrm{NaCl}$ alone or in combination with gamma rays is shown in Tab. 6 and Fig. 1. It is evident that one common band was observed under control as well as salinity and gamma rays treatments. This band becomes much intensified in salinized cowpea leaves alone or in combination with gamma rays at 25 and $50 \mathrm{mM} \mathrm{NaCl}$ as compared with control treatment. These results are in agreement with the findings of Mohamed (2005) who reported that the induction of new isozymes and the change in the acid phosphatase isozyme profile is considered to play an important role in the cellular defense against oxidative stress, caused by salt stress.

In conclusion, this study focused on the possibility to alleviate the harmful effect of salt stress by gamma irradiation treatment. Our results provide some evidences to the important functions of $\gamma$-irradiation to solve the production problems caused by high salinity which need further investigation in the future. Seed irradiation with $50 \mathrm{~Gy}$ significantly increased plant growth, photosynthetic pigments, total carbohydrate, total phenol, proline, total free 


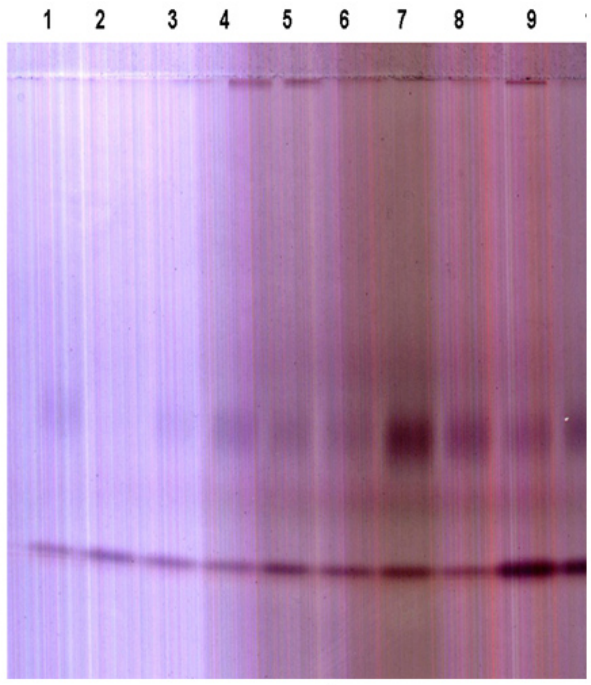

$\alpha$ - esterase

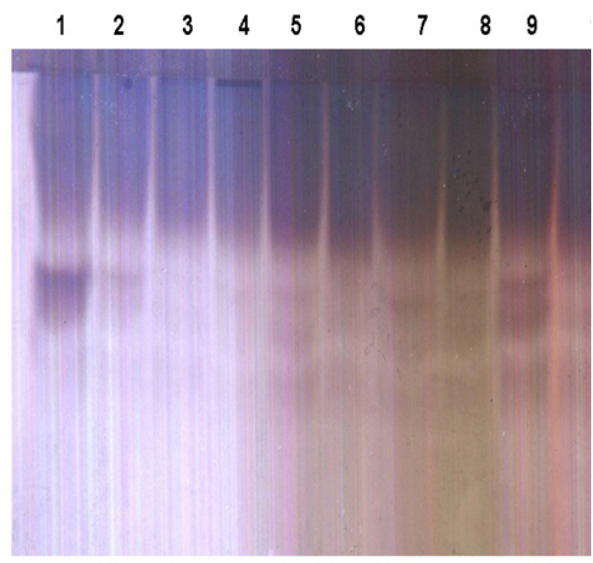

Polyphenol oxidase
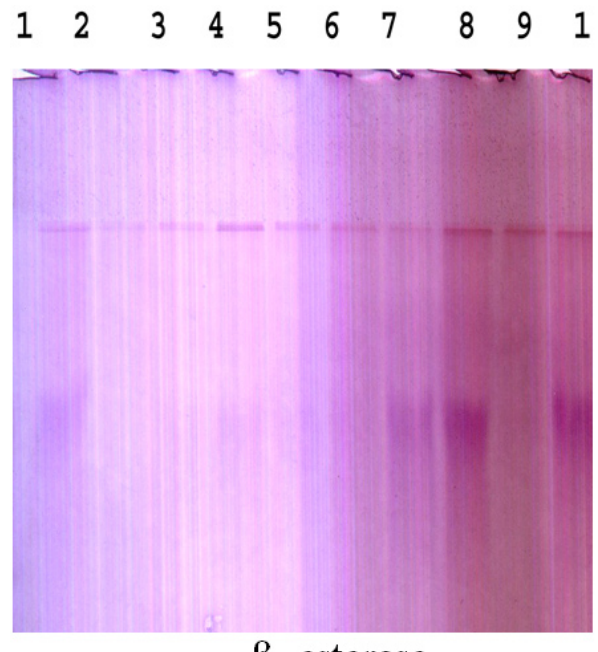

$\beta$ - esterase
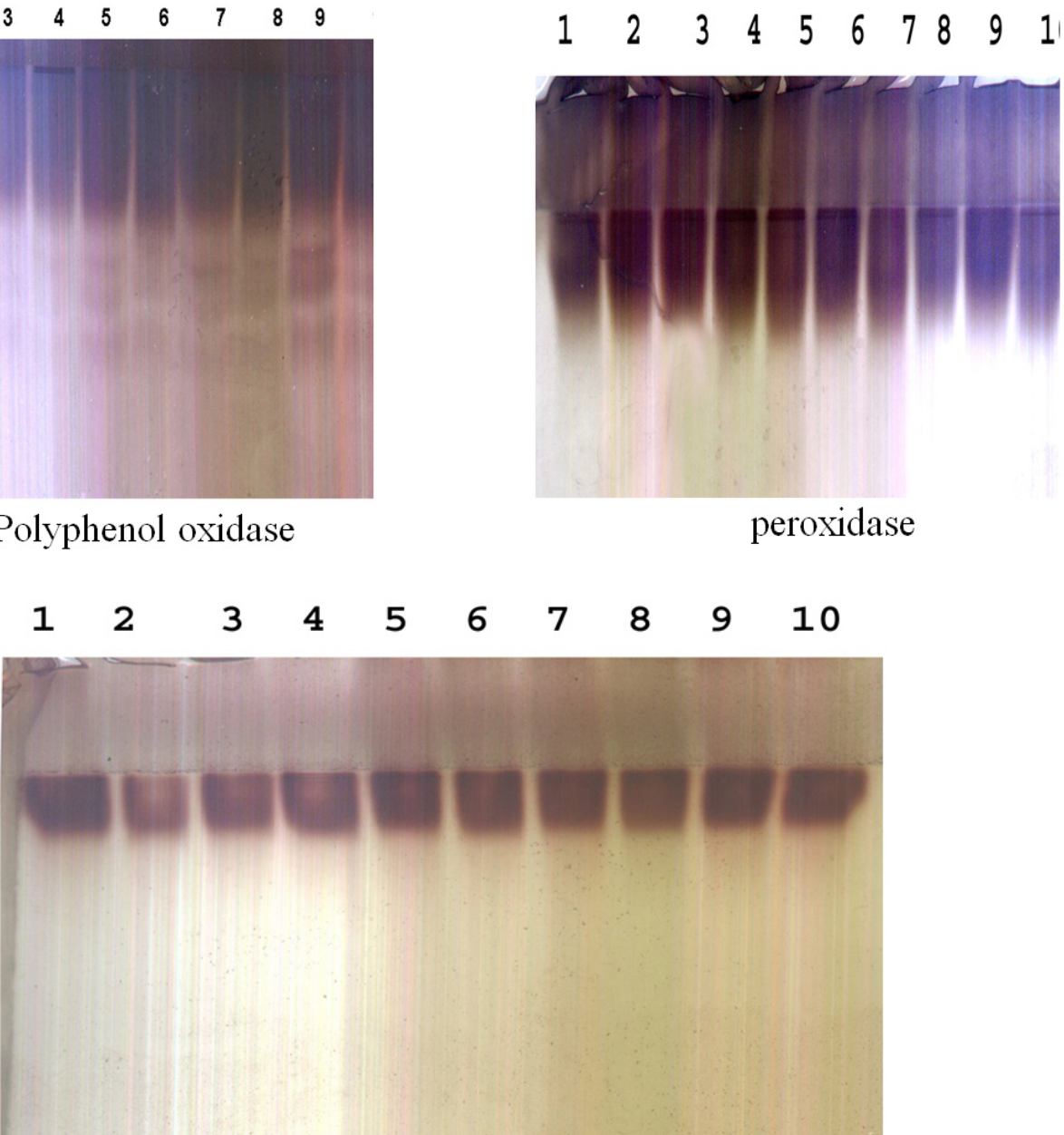

Acid phosphatase

Fig. 1. Electrophoretic patterns of $\alpha$ - and $\beta$ - esterase, polyphenol oxidase, peroxidase and acid phosphatase isozyme of cowpea leaves in response to treatment with different concentrations of $\mathrm{NaCl}$ alone or in combination with gamma ray.

1: Control; 2: 50 Gy; 3: $25 \mathrm{mM} \mathrm{NaCl} ; 4: 25 \mathrm{mM} \mathrm{NaCl}+50$ Gy; 5: $50 \mathrm{mM} \mathrm{NaCl} ; 6: 50 \mathrm{mM} \mathrm{NaCl}+50 \mathrm{~Gy} ; 7: 100 \mathrm{mM} \mathrm{NaCl} ; 8: 100$ $\mathrm{mM} \mathrm{NaCl}+50$ Gy; 9: $200 \mathrm{mM} \mathrm{NaCl} ; 10: 200 \mathrm{mM} \mathrm{NaCl}+50$ Gy 
112

amino acids and the contents of $\mathrm{N}, \mathrm{P}, \mathrm{K}^{+}, \mathrm{Ca}^{+2}$ and $\mathrm{Mg}^{+2}$ compared to non irradiated ones under salinity. On the other hand, irradiation with gamma rays decreased lipid peroxidation, $\mathrm{Na}^{+}$and $\mathrm{Cl}^{-}$contents which may contribute in part to activate processes involved in the alleviation of the harmful effect of salt. Electrophoretic studies of $\alpha$-esterase, $\beta$-esterase, polyphenol oxidase, peroxidase and acid phosphatase isozymes showed wide variations in their intensities among all treatments.

\section{References}

Afify AMR, El-Beltagi HS, Fayed SA, Shalaby EA (2011). Acaricidal activity of successive extracts from Syzygium cumini L. Skeels (Pomposia) against Tetranychus urticae Koch. Asian Pac J Trop Biomed 1(5):359-364.

Alikamanoglu S, Yaycli O, Atak C, Rzakoulieva A (2007). Effect of magnetic field and gamma radiation on Paulowinia tomentosa tissue culture. Biotechnol 21(1):129-134.

Almodares A, Hadi MR, Ahmadpour H (2008). Sorghum stem yield and soluble carbohydrates different salinity levels. African J Biotech 22:4051-4055.

Aly AA (2010). Biosynthesis of phenolic compounds and water soluble vitamins in culantro (Eryngium foetidum L.) plantlets as affected by low doses of gamma irradiation. Analele Universității din Oradea - Fascicula Biologie 2:356-361.

Aly AA, El-Beltagi HES (2010). Influence of ionizing irradiation on the antioxidant enzymes of Vicia faba L. Grasas Y Aceites 61 (3):288-294.

Amirjani MR (2011). Effect salinity stress on growth, sugar content, pigments and enzyme activity of rice. Inter J Bot $7(1): 73-81$.

AOAC (1995). Association of Official Agricultural Chemists. Official methods of analysis $16^{\text {th }} \mathrm{ed}$. Washington D.C., USA.

AOAC (2005). Association of Official Agricultural Chemists. Official Methods of Analysis, $18^{\text {th }}$ ed. Hortwitz W, Latimer GW (Ed.). AOAC-Int. Suite 500, 481 North Frederick Avenue, Gaithersburg, Maryland USA.

Arora N, Bhardwaj R, Sharma P, Arora HK (2008). Homobrassinolide alleviates oxidative stress in salt-treated maize (Zea mays L.) plants. Brazilian J Plant Physiol 20:153-157.

Ashraf MA, Ashraf M, Ali Q (2010). Response of two genetically diverse wheat cultivars to salt stress at different growth stages: leaf lipid peroxidation and phenolic contents. Pak J Bot 42(1):559-565.

Ashraf M (2009). Biotechnological approach of improving plant salt tolerance using antioxidants as markers. Biotechnol. Adv 27:84-93.

Ashraf M, Cheema AA, Rashid M, Qamar Z (2003). Effect of gamma rays on $\mathrm{M} 1$ generation in Basmati rice. Pak J Bot 35(5):791-795.

Baek MH, Chung BY, Kim JH, Wi SG, Kim JS, Lee IJ (2006). Gamma radiation and hormone treatment as tools to reduce salt stress in rice (Oryza sativa L.). J Plant Bio 49(3):257260.

Bates LS, Waldren RP, Teare LD (1973). Determination of proline for water-stress studies. Plant Soil 39:205-207.

Binzel ML, Hess FD, Bressan RA, Hasegawa PM (1988). Intracellular compartmentation of ions in salt adapted tobacco cells. Plant Physiol 86:607-614.

Bohnert HJ, Su H, Shen B (1999). Molecular mechanisms of salinity tolerance 29-60 p. In: Shinozaki K, YamaguchiShinozaki K (Eds.). Molecular responses to cold, drought, heat and salt stress in higher plants, University of Arizona, Arizona.

Bozcuk S (1970). Water and salt relations of statics species with particular reference to the problem of halophytes. Ph.D. Thesis University of Sussex, UK.

Chakravarty B, Sen S (2001). Enhancement of regeneration potential and variability by $\gamma$-irradiation in cultured cells of Scilla indica. Biologia Plant 44(2):189-193.

Dhanapackiam S, Muhammad MH (2010). Effect of salinity on chlorophyll and carbohydrate contents of Sesbania grandiflora seedlings. Indian J Sci Technol 3(1):64-66.

Dihazi AD, Jaitt F, Zouine J, Hassni ME, Hardami IE (2003). Effect of salicylic acid on phenolic compounds related to date palm resistance to Fusarium oxysporum sp. Albedimis Phytopath Medit 423:9-16.

Dubois M, Gilles KA, Hamilton JK, Rebers PA, Smith F (1956). Colorimetric method for determination of sugars and related substances. Anal Chem 28:350-356.

El-Baz FK, Mohamed AA, Aly AA (2003). Development of biochemical markers for salt stress tolerance in cucumber plants. Pak J Bio Sci 6(1):16-22.

El-Beltagi HS (2001). Biochemical studies on some Egyptian plants and its relation with environment. M. Sc. Thesis, Biochemistry Department, Faculty of Agriculture, Cairo University.

El-Beltagi HS (2004). Biochemical studies on cadmium and lead effects on radish plant. Ph. D. Thesis, Biochemistry Department, Faculty of Agriculture, Cairo University.

El-Beltagi HES, Salama ZA, El-Hariri DM (2008). Some biochemical markers for evaluation of flax cultivars under salt stress conditions. J Natural Fibers 5(4):316-330.

El-Beltagi HES (2011). Effect of roasting treatments on protein fraction profiles, some enzyme activities of Egyptian peanuts. Int J Food Sci Nutr 62(5):453-456.

El-Beltagi HS, Kesba HH, Abdel-Alim AI, Al-Sayed AA (2011a). Effect of root-knot nematode and two species of crown on antioxidant activity of grape leaves. Afr J Biotechnol 10(57):12202-12210.

El-Beltagi HS, Ahmed OK, El-Desouky W (2011b). Effect of low doses $\gamma$-irradiation on oxidative stress and secondary metabolites production of rosemary (Rosmarinus officinalis L.) callus culture. Radiat Phys Chem 80:968-976.

El-Hendawy SE, Hu Y, Yakout GM, Awad AM, Hafiz SE, 
Schmidhalter U (2004). Evaluating salt tolerance of wheat genotypes using multiple parameters. European J Agron 22:245-253.

El-Sayed OE, Rizkalla AA, Sabri SRS (2007). In vitro mutagenesis for genetic improvement of salinity tolerance in wheat. Res J Agri Bio Sci 4(5):377-383.

Esfandiari E, Shakiba MR, Mahboob SA, Alyari H, Shahabivand $S$ (2008). The effect of water stress on antioxidant content, protective enzyme activities, proline content and lipid peroxidation in wheat seedling. Pak J Biol Sci 11(15):1916 1922.

Foyer CH, Noctor G (2002). Oxygen processing in photosynthesis: regulation and signaling. New Phytol 146:359-388.

Greenway H, Munns R (1980). Mechanisms of salt tolerance in nonhalophytes. Ann Rev Plant Physiol 31:149-190

Hameed A, Shah TM, Atta BM, Haq MA, Sayed H (2008a). Gamma irradiation effects on seed germination and growth, protein content, peroxidase and protease activity, lipid peroxidation in desi and kabuli chickpea. Pak J Bo 40(3):10331041.

Hameed A, Naseer S, Iqbal T, Sayed H, Ahsanul M (2008b). Effect of $\mathrm{NaCl}$ salinity on seedling growth, senescence, catalase and protease activities in two wheat genotypes differing in salt tolerance. Pak J Bot 40(3):1043-1051.

Hassanein AM (1999). Alteration in protein and esterase of peanut in response to salinity stress. Biologia Plant 42:241248.

Heath RL, Packer L (1968). Photoperoxidation in isolated chloroplasts. I. Kinetics and stoichiometry of fatty acid peroxidation. Arch Bioch Bioph 125(1):189-198.

Hernandez JA, Almansa MS (2002). Short-term effects of salt stress on antioxidant systems and leaf water relations of pea leaves. Physiol Plant 115:251-257.

Khan MH, Singha LB, Panda SK (2002). Changes in antioxidant levels in Oryza sativa L. roots subjected to $\mathrm{NaCl}$-salinity stress. Acta Physiol Plant 24:145-148.

Khan MH, Panda SK (2008). Alterations in root lipid peroxidation and antioxidative responses in two rice cultivars under $\mathrm{NaCl}$-salinity stress. Acta Physiol Plant 30:91-89.

Kiong A, Ling Pick A, Grace Lai SH, Harun AR (2008). Physiological responses of Orthosiphon stamineus plantlets to gamma irradiation. Am-Eurasian J Sustain Agric 2(2):135-149.

Kobeasy MI, El-Beltagi HS, El-Shazly MA, Khattab EAH (2011). Induction of resistance in Arachis hypogaea L. against Peanut Mottle Virus by nitric oxide and salicylic acid. Physiol Mol Plant Pathol 76:112-118.

Kovacs E, Keresztes A (2002). Effect of gamma and UV-B/C radiation on plant cell. Micron 33:199-210.

Larsen AL, Benson WC (1970). Variety specific variants of oxidative enzymes from soybean seeds. Crop Sci 10:493-495.

Lobato AKS, Oliveira Neto CF, Costa RCL, Santos Filho BG, Cruz FJR, Laughinghouse IV (2008). Biochemical and physiological behavior of Vigna unguiculata (L.) Walp. un-
113

der water stress during the vegetative phase. Asian J Plant Sci 7:44-49.

Mahajan S, Tuteja N (2005). Cold, salinity and drought stresses. Arch Bioch Bioph 444:139-158.

Mittler R (2002). Oxidative stress, antioxidants and stress tolerance. Trends Plant Sci 7:405-410.

Mohamed AA (2005). Two-dimensional electrophoresis of soluble proteins and profile of some isozymes isolated from maize plant in response to $\mathrm{NaCl}$. Res J Agri Bio Sci 1(1):3844.

Mohamed AA, El-Beltagi HS, Rashed MM (2009). Cadmium stress induced change in some hydrolytic enzymes, free radical formation and ultrastructural disorders in Radish plant. Electron J Environ, Agric Food Chem 8(10):969-983.

Mohamed HI, Gomaa EZ (2012). Effect of plant growth promoting Bacillus subtilis and Pseudomonas fluorescens on growth and pigment composition of radish plants (Raphanus sativus) under $\mathrm{NaCl}$ stress. Photosynthetica 50(2):263-272.

Mohamed HI (2011). Molecular and biochemical studies on the effect of gamma rays on lead toxicity in cowpea (Vigna sinensis) Plants. Biol Trace Elem Res 144:1205-1218.

Moore S, Stein WH (1954). A Modified ninhydrin reagent for the photometric determination of amino acids and related compounds. J Biol Chem 211:907-913.

Moussa HR (2011). Low dose of gamma irradiation enhanced drought tolerance in soybean. Acta Agronomica Hungarica 59(1):1-12.

Munnas RC (2002). Comparative physiology of salt and water stress. Plant Cell Environ 25:239-250.

Nassar AH, Hashim MF, Hassan NS, Abo-Zaid H (2006). Effect of gamma irradiation and phosphorus on growth and oil production of chamomile (Chamomilla recutita $\mathrm{L}$. Rauschert). Int J Agri Biol 6 (5):776-780.

Niu X, Bressan RA, Hasegawa PM, Pardo JM (1995). Ion homeostasis in $\mathrm{NaCl}$ stress environments. Plant Physiol 109:735-742.

O'Kane DV, Gill PB, Burdon R (1996). Chilling, oxidative stress and antioxidant response in Arabidopsis thaliana callus. Planta 198:371-377.

Rashid SUM, Asad S, Zafar Y, Washeed RA (2001). Use of radiation and in vitro techniques for development of salt tolerant mutants in sugarcane and potato. IAEA-TECDOC 1227:51-60.

SAS-Programme (1982). SAS user,s Guide Statistics SAS Institute, INC, Raleiegh. NC. 584.

Sato M, Hasegawa M (1976). The latency of spinach chloroplast phenolase. Phytochem 15:61.

Scandalios JG (1964). Tissue specific isozyme variation in maize. Heredity 55:281-285.

Shereen A, Ansari R, Mumtaz S, Bughio HR, Mujtaba SM, Shirazi MU, Khan MA (2009). Impact of gamma irradiation induced changes on growth and physiological responses of rice under saline conditions. Pak J Bot 41(5):2487-2495. 
114

Silva EC, Nogueira RJM, Araujo FP, Melo NF, Azevedo Neto AD (2008). Physiological responses to salt stress in young umbu plants. Env Exp Botany 63:147-157.

Stepien P, Klobus G (2006). Water relations and photosynthesi in Cucumis sativus L. leaves under salt stress. Biologia Plant 50(40):610-616.

Taiz L, Zeiger E (2006). Plant Physiology ( $4^{\text {th }}$ edn). Sinauer Associates, Inc., Sunderland, Massachusetts.

Vernon LP, Seely GR (1966). The chlorophylls. Academic Press. New York.

Wendel JF, Weeden JF (1989). Visualization and interpretation of plant isozymes $18 \mathrm{p}$. In: Soltis DE, Soltis PS (Eds.). Isozymes in Plant Biology, Chapman and Hall Publishers, London.
Wi SG, Chung BY, Kim JS, Kim JH, Baek MH, Lee JW, Kim YS (2007). Effects of gamma irradiation on morphological changes and biological responses in plants. Micron 38:553564.

Wise RR, Naylor AW (1987). Chilling-enhanced peroxidation: the peroxidative destruction of lipids during chilling injury to photosynthesis and ultrastucture. Plant Physiol 83:227272.

Yeo AR (1998). Molecular biology of salt tolerance in the context of whole-plant physiology. J Exp Bot 49:915-929. 